\title{
RESULTS ON THE INTERACTION OF AN INTENSE BUNCHED ELECTRON BEAM WITH RESONANT CAVITIES AT 35 GHZ
}

\author{
J. Gardelle, T. Lefevre, J.L. Rullier, C. Vermare \\ CEA/Centre d'Etudes Scientifiques et Techniques d'Aquitaine, B.P. 2, 33114 Le Barp, France \\ W. Wuensch \\ Laboratoire Européen pour la Physique des Particules (CERN), CH-1211 Genève 23, Suisse \\ S. Lidia* \\ E.O. Lawrence Berkeley National Laboratory, Berkeley, California 94720, USA \\ G.A. Westenskow* \\ Lawrence Livermore National Laboratory, Livermore, California 94551, USA \\ J.T. Donohue \\ Centre d'Etudes Nucléaires de Bordeaux-Gradignan, B.P. 120, 33175 Gradignan, France \\ Y. Meurdesoif \\ Laboratoire de Physique Théorique (LPT), Université de Bordeaux I, 33400 Talence, France \\ J.M. Lekston \\ CEA Centre d'Etudes d'Ile de France (CIF), DPTA/SPPE, BP 12, 91680 Bruyères-le-Châtel, France
}

\begin{abstract}
The Two-Beam Accelerator (TBA) concept is currently being investigated both at Lawrence Berkeley National Laboratory (LBNL) and at CERN. As part of this program, a $7 \mathrm{MeV}, 1-\mathrm{kA}$ electron beam produced by the PIVAIR accelerator at CESTA has been used to power a free electron laser (FEL) amplifier at $35 \mathrm{GHz}$. At the FEL exit, the bunched electron beam is transported and focused into a resonant cavity built by the CLIC group at CERN. The power and frequency of the microwave output generated when the bunched beam traverses two different cavities are measured.
\end{abstract}

\section{INTRODUCTION}

In order to attain linear collider energies of order $1 \mathrm{TeV}$, it is desirable to develop efficient high-power microwave sources operating in the frequency range of $10-30 \mathrm{GHz}$, or even higher. In this context, the TBA [1] is being studied both at LBNL with the 11.4-GHz prototype RTA [2] and at CERN with the Compact Linear Collider (CLIC) project at $30 \mathrm{GHz}$ [3]. In the TBA, the intense low-energy electron drive beam is bunched at the desired operating frequency and, upon passing through resonant cavities, it generates RF power to drive the high gradient accelerating structures of the main beam. An important issue of the TBA concerns the production of the bunched drive beam. At CESTA we have been studving the use of

*Work in the U.S. was performed under the auspices of the U.S. Department of Energy under contracts AC03-76SF00098 (LBNL) and W-7405-ENG-48 (LLNL). a FEL as a possible source of an intense bunched beam. We have observed and characterized the bunching which occurs in an induction linac-driven FEL both in amplifier [4] and in Self Amplified Spontaneous Emission (SASE) modes [5]. We report here on the recent results of an experiment which used the PIVAIR induction linac. Two resonant cavities designed by LBNL and built by CERN have been tested with the bunched beam exiting the wiggler of a $35 \mathrm{GHz}$ amplifier. We describe here the results of this experiment.

\section{EXPERIMENTAL SET UP}

\subsection{The PIVAIR induction linac}

PIVAIR is the prototype of the AIRIX accelerator which is under construction in France for flash X-ray radiography [6]. The injector, developed by Pulse Sciences Inc. (PSI), delivers a single-shot, $3.6 \mathrm{MeV}, 3$ $\mathrm{kA}, \quad 60 \mathrm{~ns}$ FWHM electron beam. The beam is subsequently accelerated to $7 \mathrm{MeV}$ by 16 induction cells. The first 8 cells use rexolite gap insulators and are fed by $180 \mathrm{kV}$ pulses coming from 4 external high voltage generators. The last 8 cells, working under vacuum with no gap insulators, are less sensitive to breakdown problems and are driven by $240 \mathrm{kV}$ pulses. Inside each cell-block there are a solenoid and two steerers which are used to transport the beam to the accelerator exit. Beam 
Position Monitors are installed every 4 induction blocks in order to monitor beam current and centroid position. PIVAIR has an excellent shot-to-shot reproducibility. The normalized edge emittance at the injector exit is 1000 $\pi . \mathrm{mm} . \mathrm{mrad}$ and the energy spread is less than $1 \%$ over 60 ns.

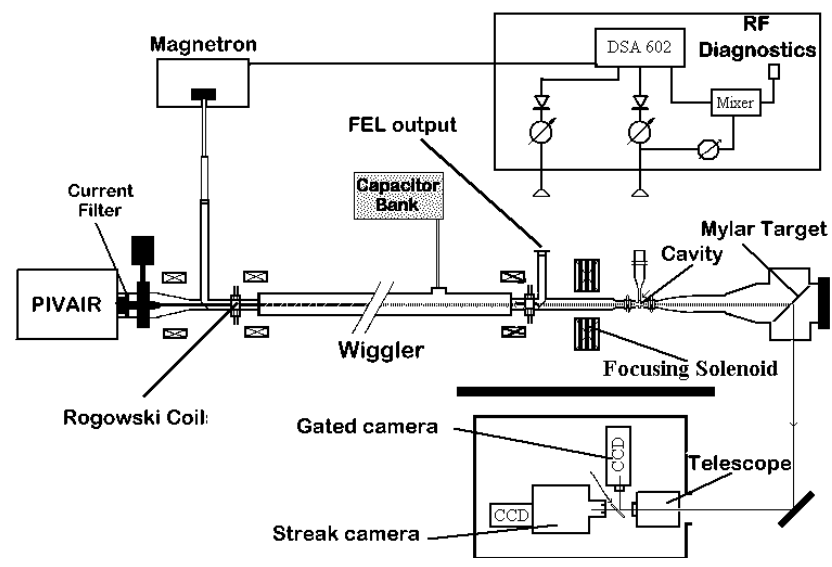

Figure 1: Layout of the experiment.

\subsection{The FEL experiment}

In figure 1, we show the overall experiment and Table 1 gives the main FEL parameters, PIVAIR is schematized at left. Immediately after the accelerator exit a current filter is used to reduce the beam current to $1 \mathrm{kA}$. Then, two solenoids match the beam into the pulsed helical wiggler of the FEL. A $35 \mathrm{GHz}$ electromagnetic signal, produced by a magnetron, is injected into the wiggler and is amplified in the wiggler by the electron beam. As a consequence of the FEL mechanism, the beam bunches during its passage through the wiggler. A suitable magnetic profile at the wiggler entrance is necessary to obtain an adiabatic injection giving rise to the correct trajectories in the wiggler. Similarly, an adiabatic exit is needed to extract the beam from the wiggler on the axis. Upon leaving the FEL, the bunched beam is focused by a set of 4 thick coils which generate a magnetic field up to $2700 \mathrm{G}$ in order to produce a narrow beam waist at the position of the RF cavity. The coils can be moved along the longitudinal axis to optimize the waist size. The latter is measured by the Cerenkov diagnostic and it must be as small as possible. Alignment is very critical and can be changed by moving the coils transversely or by tilting them. First, we use a dummy which simulates the cavity longitudinal dimensions. It permits us to adjust beam alignment by measuring the transmitted current. The FEL signal is deflected out of beam line by a wire mesh. FEL power and frequency measurements are performed using standard Ka-band waveguides, horns, attenuators and calibrated Shottky diodes. Rogowski coils are used to measure the beam current at different axial positions. Beam size and position are obtained by a gated camera which collects a small part of the Cerenkov emission produced when the beam passes trough a aluminizedmylar target. A fast streak camera is used to measure beam bunching.

Table 1: Main FEL parameters

\begin{tabular}{|lc|}
\hline Beam energy & $7 \mathrm{MeV}$ \\
Beam current & $1 \mathrm{kA}$ \\
Wiggler period & $20 \mathrm{~cm}$ \\
Wiggler length & $640 \mathrm{~cm}$ \\
Wiggler resonant field & $1650 \mathrm{G}$ \\
Beam pipe radius & $19 \mathrm{~mm}$ \\
Frequency & $35.04 \mathrm{GHz}$ \\
Input power & $5 \mathrm{~kW}$ \\
\hline
\end{tabular}

\subsection{The cavities}

Two pill-box cavities of length $1 \mathrm{~mm}$, were designed by LBNL and built by CERN (figure 2). The internal diameter of the $11 \mathrm{~mm}$-long pipe where the beam propagates in is $4 \mathrm{~mm}$. In order to match to the main 38 $\mathrm{mm}$ internal diameter beam pipe, a smooth tapering in radius was necessary.

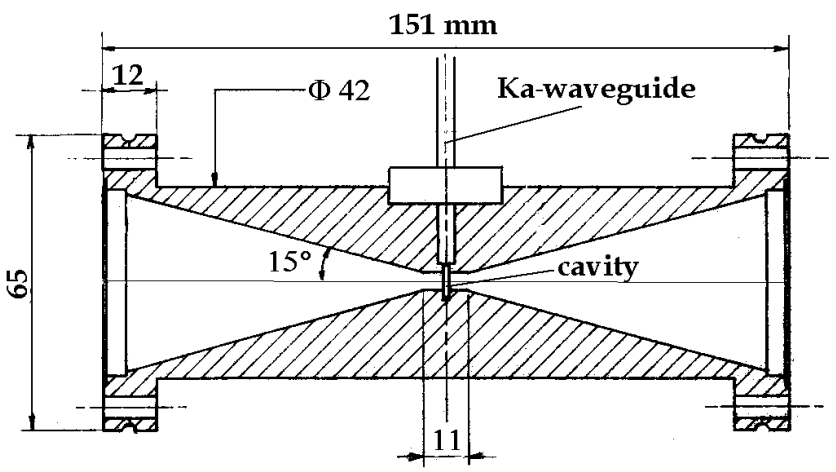

Figure 2: Drawing of the $35 \mathrm{GHz}$ pill-box cavity support

The two cavities differ mainly by the size of their output ports. The port is attached to a connecting waveguide which is expanded to mate with standard WR28 rectangular guide. Their resonant frequencies and $\mathrm{Q}$ values are different in order to obtain two couplings [7]. The low-Q cavity has a radius of $3.92 \mathrm{~mm}$ whereas the medium-Q cavity has a radius of $3.8 \mathrm{~mm}$. The latter is slightly detuned from $35 \mathrm{GHz}$ in order to study the effects of the detuning on the output power as well as on the beam bunching. 


\section{EXPERIMENTAL RESULTS}
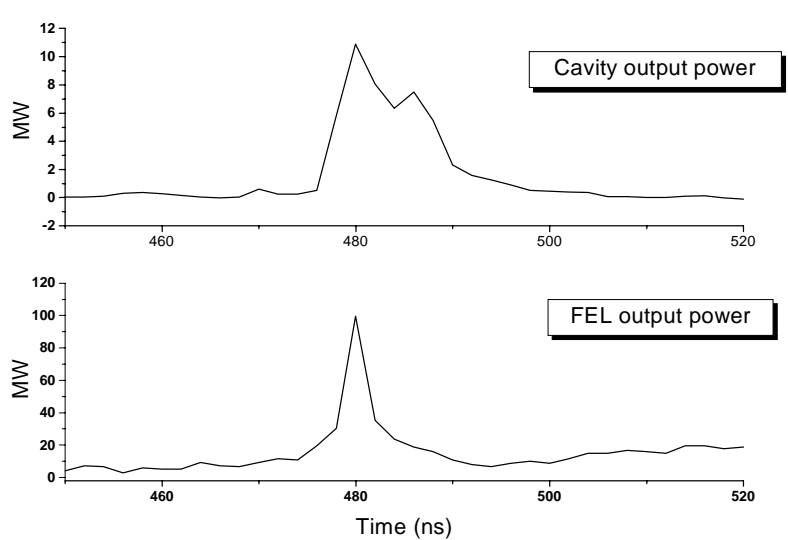

Figure 3: Example of the output signal from the low-Q tuned cavity and the coresponding FEL power

The injected RF signal coming from the magnetron was amplified by the FEL up to $100 \mathrm{MW}$ at a measured frequency of $35.04 \mathrm{GHz}$. An important beam loss occurred in the wiggler. The Rogowski coil at the wiggler exit gives a value of about $300 \mathrm{~A}$ when $800 \mathrm{~A}$ are measured at the wiggler entrance. Nevertheless, the bunched beam was extracted from the adiabatic exit and focused to a $8-\mathrm{mm}$ diameter waist by the four-coil module running at its maximum current of $200 \mathrm{~A}$. Since the aperture of the cavities is only $4 \mathrm{~mm}$, an additional loss of beam current occured when introducing the beam. In figure 3, we display the output power from the low-Q cavity as well as the corresponding FEL signal. A microwave signal with a peak power of $12 \mathrm{MW}$ was obtained in the output port of the cavity, whose frequency coincides, within errors, with that of the magnetron. With the medium-Q cavity, slightly detuned in frequency, the measured power is of order $700 \mathrm{~kW}$ at $35.6 \mathrm{GHz}$ as shown in figure 4 . In general the current value $10-\mathrm{cm}$ upstream from the cavity is $250 \pm 30 \mathrm{~A}$, while at the exit the current is $120 \pm 20 \mathrm{~A}$.

The output power in the detuned cavity is less than what we obtained with the tuned cavity, as predicted by by the simulations [7]. The measurements of the frequency of the signals exiting the cavities are in very good agreement with the measurements of their "cold" resonances (35.2 $\mathrm{GHz}$ for the low-Q and $35.7 \mathrm{GHz}$ for the medium-Q) which were obtained without the presence of the electron beam by using a network analyzer.

\section{CONCLUSION}

We have described here the results of an experiment which constitutes a first proof of principle of microwave power extraction from a bunched electron beam in simple a high frequency RF-cavity working at $35 \mathrm{GHz}$. At this
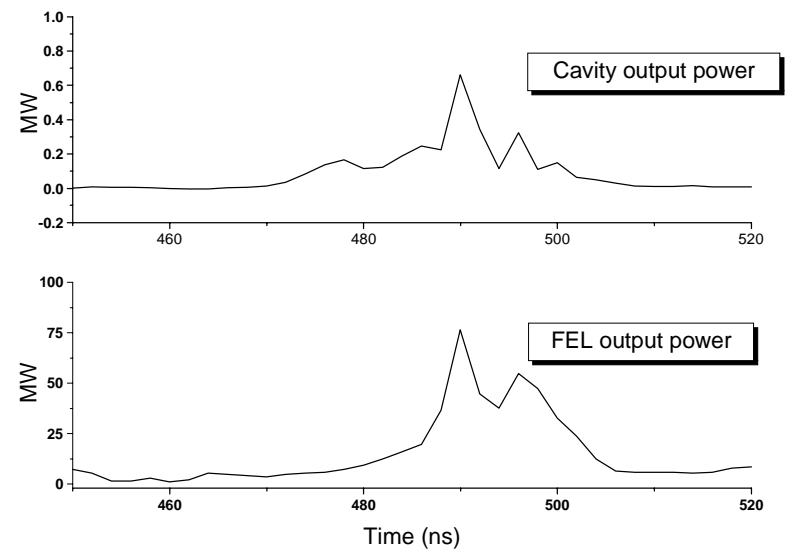

Figure 4: Example of the output signal from the medium-

Q detuned cavity and the coresponding FEL power

time, we have not finished analyzing all the data but we

believe that an improved version of our experiment would be able to achieve a net increase of the output power from the cavity.

\section{REFERENCES}

[1] A.M. Sessler, The Laser Accel. of Part., AIP Conf. Proc. N ${ }^{\circ} 91$ (American Institute of Physics, New York, 1982), p. 151.

[2] G.A. Westenskow, et. al., Proc. of LINAC'98, Chicago, (1998).

[3] J.P. Delahaye, et. al.., Proc. of VII International Workshop on Linear Colliders, Zvenigorod, Russia, (1997).

[4]] J. Gardelle, J. Labrouche, J.L. Rullier, Phys. Rev. Lett. 76, 4532 (1996). J. Gardelle, et. al.., Phys. Rev. Lett. 79, 3905 (1997).

[5] T. Lefevre, et. al., Phys. Rev. Lett. 82, 323 (1999).

[6] P. Anthouard, et. al., Proc. of the Part. Acc. Conf., Vancouver, Canada, (1997).

[7] S. Lidia , et. al., these proceedings. 